\title{
Identification and Nonlinear PID Control of Hammerstein Model using Polynomial Structures
}

\author{
Zeineb RAYOUF \\ Advanced System Laboratory, \\ Polytechnic School of Tunisia \\ BP 743, 2078 \\ La Marsa, Tunisia
}

\author{
Chekib GHORBEL \\ Advanced System Laboratory, \\ Polytechnic School of Tunisia \\ BP 743, 2078 \\ La Marsa, Tunisia
}

\author{
Naceur BENHADJ BRAIEK \\ Advanced System Laboratory, \\ Polytechnic School of Tunisia \\ BP 743, 2078 \\ La Marsa, Tunisia
}

\begin{abstract}
In this paper, a new nonlinear discrete-time PID is proposed to control Hammerstein model. This model is composed by a static nonlinearity gain associated to a linear dynamic subsystem. Nonlinear polynomial structures are used to identify and to control this class of systems. The determination of parameters is based on the use of RLS algorithm. A coupled two-tank process is given to illustrate the effectiveness of the proposed approach.
\end{abstract}

Keywords-Parametric identification; Hammerstein model; RLS algorithm; Polynomial structure; Nonlinear PID controller

\section{INTRODUCTION}

There are well-developed theories for the control and identification of linear time invariant (LTI) systems. In modern applications, physical systems are nonlinear. This drives an increasing need for modeling techniques able to adequately describe these systems behavior. Nonlinear system identification is an important tool wish can be used to improve control performance. Indeed, there are serval types models that describe perfectly this process such as Hammerstein model [1], polynomial structures [2] [3], voltera [4], NARMAX [5], etc.

Hammerstien model is consisted of a static nonlinearity followed by a linear dynamic system. Many chemical processes have been modeled with it, for examples, $\mathrm{pH}$ neutralization processes [6], distillation columns [7] [8], polymerization reactor [9] [10] and dryer process [11].

Polynomial models are possibly the most attractive of all nonlinear representations due to the inherent simplicity of the model structure and because they revealed the dynamical properties of the underlying system is a very straightforward manner [12].

Serval nonlinear predictive control algorithms are existed based on PID [13], neural networks [14], B-spline neural networks [15], Fuzzy logic [16] adaptive predictive control [17] [18]. In most algorithms for nonlinear predictive control, their performance functions are minimized using nonlinear programming techniques to compute the future manipulated variables in on-line optimization. This can make the realization of the algorithms very difficult for real-time control.

An important advantage of block-structured models is that they allow the use of standard linear controller design methods. This is possible because static nonlinearity in the process can be negated by inserting the nonlinear inverse of static nonlinearity at the appropriate place in the loop [19] [20] [21].
For the Hammerstein model, reverse nonlinear tuning must be placed at the output of the controller, wish only sees the linear dynamic part of the process and conventional linear controller methods can be used. Often static nonlinearity may be non-invertible this present a limit for this method.

In this work, a polynomial structure is employed to describe the nonlinear static function of Hammerstein model. Recursive least square RLS algorithm is used to estimate the unknown parameters. A new nonlinear discrete PID is proposed. It is composed by a linear controller associated with the inverse of the nonlinearity wish is obtained by an approximation using polynomial structure.

The remainder of this paper is organized as follows: first, a Parametric identification of the Hammerstein model is defined. Second, a proposed nonlinear polynomial structure of Hammerstein model is described. Third, a method to control the model is presented. After that, the proposed identification and control method are applied to a coupled two-tank system.

\section{PARAmetric Identification OF MOdel HAMMERSTEIN}

Assume that the Hammerstein model of Fig. 1 is composed of a nonlinear block $f($.$) associated with a linear sub-system$ $\frac{B\left(q^{-1}\right)}{A\left(q^{-1}\right)}$. It is described by:

$$
\left\{\begin{array}{l}
y_{k}=\frac{B\left(q^{-1}\right)}{A\left(q^{-1}\right)} v_{k} \\
v_{k}=f\left(u_{k}\right)
\end{array}\right.
$$

with:

$$
\begin{gathered}
A\left(q^{-1}\right)=1+a_{1} q^{-1}+\ldots+a_{n A} q^{-n_{A}} \\
B\left(q^{-1}\right)=b_{1} q^{-1}+\ldots+b_{n B} q^{-n_{B}} \\
v_{k}=d_{1} u_{k}+d_{2} u_{k}^{2}+\ldots+d_{N} u_{k}^{N}
\end{gathered}
$$

$q^{-1}$ delay operator, $u_{k}$ input of the system, $y_{k}$ output, $v_{k}$ the unmeasurable internal signal and $w_{k}$ represents the modeling error, external disturbances, etc.

In order to have a unique parameterization of the Hammerstein model structure, the first coefficient of the nonlinear function $f($.$) equals to 1, d_{1}=1$, [22] [23]. 


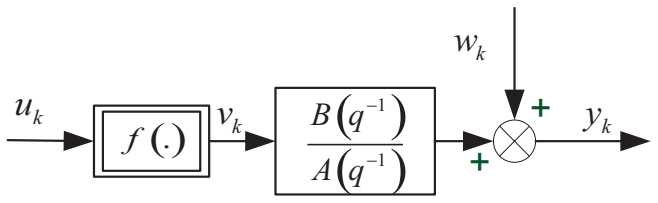

Fig. 1 - Hammerstein model structure.

The output $y_{k}$ is given by:

$$
\begin{aligned}
y_{k}= & -\sum_{i=1}^{n_{a}} a_{i} y_{k-i}+ \\
& \sum_{i=1}^{n_{b}} b_{i}\left(u_{k-i}+\sum_{p=2}^{N} d_{p} \mathrm{u}_{k-i}^{p}\right)
\end{aligned}
$$

Eq. 2 can be put in the following form:

$$
y_{k}=\Phi_{k}^{T} \theta_{k}
$$

with:

$$
\begin{gathered}
\Phi_{k}=\left(\begin{array}{c}
Y_{k} \\
U_{k}
\end{array}\right), \theta_{k}=\left(\begin{array}{c}
a_{k} \\
b_{k} \\
s_{k}
\end{array}\right), \\
Y_{k}=\left(\begin{array}{c}
-y_{k-1} \\
-y_{k-2} \\
\vdots \\
-y_{k-n_{a}}
\end{array}\right), U_{k}=\left(\begin{array}{c}
U_{1 k} \\
U_{2 k} \\
\vdots \\
U_{N k}
\end{array}\right), \\
U_{j k}=\left(\begin{array}{c}
u^{j}{ }_{k-1} \\
u^{j}{ }_{k-2} \\
\vdots \\
u^{j}{ }_{k-n_{b}}
\end{array}\right), \text { for } j=1,2, \ldots, N \\
a_{k}=\left(\begin{array}{c}
b_{1 k} \\
b_{1 k} \\
a_{2 k} \\
\vdots \\
a_{n k}
\end{array}\right), b_{k}=\left(\begin{array}{c}
d_{2 k} b_{k} \\
d_{3 k} b_{k} \\
\vdots \\
b_{n k}
\end{array}\right),
\end{gathered}
$$

$\Phi_{k}$ and $\theta_{k} \in R^{n_{R}}$ where $n_{R}=n_{a}+N n_{b}, Y_{k} \in R^{n_{a}}$, $U_{k} \in R^{N n_{b}}, U_{j k} \in R^{n_{b}}, a_{k} \in R^{n_{a}}, b_{k} \in R^{n_{b}}$ and $s_{k} \in R^{N n_{b}}$.

The parameter vector $\theta_{k}$ can be estimated using the RLS algorithm. It is described by the following equations:

$$
\left\{\begin{array}{l}
\hat{\theta}_{k}=\hat{\theta}_{k-1}+P_{k} \Phi_{k} \varepsilon_{k} \\
P_{k}=P_{k-1}-\frac{P_{k-1} \Phi_{k} \Phi_{k}^{T} P_{k-1}}{1+\Phi_{k}^{T} P_{k-1} \Phi_{k}} \\
\varepsilon_{k}=y_{k}-\hat{\theta}_{k-1}^{T} \Phi_{k}
\end{array}\right.
$$

where $P_{k}$ is the adaptation gain matrix, $\Phi_{k}$ is the observation vector and $\theta_{k}$ is the parameters vector.

\section{Proposed Nonlinear Polynomial StRUCTURE OF THE HAMMERSTEIN MODEL}

We propose a nonlinear polynomial structure of $f($.$) . It's$ described by :

$$
v_{k}=f\left(u_{k}\right)=\sum_{i=1}^{r} w_{i, k} U_{k}^{[i]}=\sum_{i=1}^{r} \tilde{w}_{i, k} \tilde{U}_{k}^{[i]}
$$

with $w_{i, k}, i=1,2, \ldots, r \quad \in \quad R^{1 \times n_{i}} \quad$ (resp. $\left.\tilde{w}_{i, k} \in R^{1 \times n_{i}}\right)$ are variable vector and $U_{k}=$ $\left(u_{k}, u_{k-1}, \ldots, u_{k-n_{u}+1}\right)^{T} \in R^{n_{u}}$ where $n_{u} \leq n_{A}$.

$U_{k}^{[i]}$ is the Kronecker power of the vector $U_{k}$ defined as [12]:

$\left\{\begin{array}{l}U_{k}^{[0]}=1 \\ U_{k}^{[i]}=U_{k}^{[i-1]} \otimes U_{k}=U_{k} \otimes U_{k}^{[i-1]}, \text { for } i \geq 1\end{array}\right.$

$\otimes$ designates the symbol of the Kroneker product,

$$
\tilde{U}_{k}^{[i]} \in R^{n_{i}}, \text { for } i=1,2, \ldots, r \text { and } n_{i}=\left(\begin{array}{c}
n+i-1 \\
i
\end{array}\right),
$$

is the non-redundant. It's defined as:

$$
\left\{\begin{array}{c}
\tilde{U}_{k}^{[1]}=U_{k}^{[1]}=U_{k} \\
\tilde{U}_{k}^{[i]}=\left(\begin{array}{l}
u_{k}^{i} \\
u_{k}^{i-1} u_{k-1} \\
\vdots \\
u_{k}^{i-1} u_{k-n} \\
\vdots \\
u_{k}^{i-2} u_{k-n}^{2} \\
\vdots \\
u_{k}^{i-3} u_{k-1}^{3} \\
\vdots \\
u_{k-n}^{i}
\end{array}\right), \text { for } i \geq 2
\end{array}\right.
$$

when the repeated components of the redundant $(i t h-$ power) $U_{K}^{[i]}$ are omitted and $r$ is the polynomial order.

In this work, we have modeled $v_{k}=f\left(u_{k}\right)$ by a nonlinear polynomial structure as:

$$
v_{k}=\tilde{w}_{1, k}^{1} U_{k}+\tilde{w}_{2, k}^{1} \tilde{U}_{k}^{[2]}+\tilde{w}_{3, k}^{1} \tilde{U}_{k}^{[3]}+O\left(U_{k}^{[4]}\right)
$$

with $U_{k}=\left(\begin{array}{l}u_{k} \\ u_{k-1}\end{array}\right), \quad \tilde{w}_{1, k}^{1}=\left(\begin{array}{ll}1 & 1\end{array}\right)$, $\tilde{w}_{2, k}^{1}=\left(\begin{array}{lll}\alpha_{1, k} & \alpha_{2, k} & \alpha_{3, k}\end{array}\right) \quad$ and $\quad \tilde{w}_{3, k}^{1}=$ $\left(\begin{array}{llll}\alpha_{4, k} & \alpha_{5, k} & \alpha_{6, k} & \alpha_{7, k}\end{array}\right)$

and the linear dynamic system by:

$y_{k}=-a_{1, k} y_{k-1}-a_{2, k} y_{k-2}+b_{1, k} v_{k-1}+b_{2, k} v_{k-2}$

Equations 8 and 9 give:

$$
\begin{aligned}
& y_{k}=-a_{1, k} y_{k-1}-a_{2, k} y_{k-2}+\tilde{w}_{1, k}^{2} U_{k-1} \\
& +\tilde{w}_{2, k}^{2} \tilde{U}_{k-1}^{[2]}+\tilde{w}_{3, k}^{2} \tilde{U}_{k-1}^{[3]}+O\left(U_{k}^{[4]}\right)
\end{aligned}
$$


with:

$$
\left\{\begin{aligned}
\tilde{w}_{1, k}^{2}= & \left(b_{1, k} ; b_{1, k}+b_{2, k}\right)^{T} \\
\tilde{w}_{2, k}^{2}= & \left(b_{1, k} \alpha_{1, k} ; b_{1, k} \alpha_{2, k} ; b_{1, k} \alpha_{3, k}+b_{2, k} \alpha_{1, k}\right)^{T} \\
\tilde{w}_{3, k}^{2}= & \left(b_{1, k} \alpha_{4, k} ; b_{1, k} \alpha_{5, k} ; b_{1, k} \alpha_{6, k} ;\right. \\
& \left.b_{1, k} \alpha_{7, k}+b_{2, k} \alpha_{4, k}\right)^{T}
\end{aligned}\right.
$$

Eq. 10 can be written in the form (3) with:

$$
\Phi_{k}=\left(\begin{array}{c}
-y_{k-1} \\
-y_{k-2} \\
U_{k-1} \\
\tilde{U}_{k-1}^{[2]} \\
\tilde{U}_{k-1}^{[3]}
\end{array}\right) \text { and } \theta_{k}=\left(\begin{array}{c}
a_{1, k} \\
a_{2, k} \\
\tilde{w}_{1, k}^{2} \\
\tilde{w}_{2, k}^{2} \\
\tilde{w}_{3, k}^{2}
\end{array}\right)
$$

The parameters $\alpha_{i, k}, \quad i=1,2, \ldots, 7$, will be successively estimated by the RLS algorithm.

\section{Nonlinear Pid Controller of the HAMMERSTEIN MODEL}

In this section, the control of the Hammerstein model with a nonlinear PID will be discussed. Firstly, we presented a nonlinear PID based on the exact inverse of $f($.$) . After that, we$ proposed a method to determine the inverse of the nonlinearity using the polynomial structure wish will be used to control the Hammerstein model.

\section{A. Nonlinear PID controller using the exact inverse}

The design strategy discrete-time control is implemented by introducing the inverse function of Hammerstein model [20]. Fig. 2. illustrates the control of Hammerstein model. It is based PID regulator as [24]:

$$
u_{k}=K_{p} \varepsilon_{k}+K_{i} T_{e} \sum_{j=0}^{k} \varepsilon_{j}+K_{d} \frac{\varepsilon_{k}-\varepsilon_{k-1}}{T_{e}}
$$

where $\varepsilon_{k}=y_{k}^{c}-y_{k}^{m}$ is the error, $y_{k}^{c}$ is the set point, $y_{k}^{m}$ is the response of the model, $T_{e}$ is the sampling period and $K_{p}, K_{i}$ and $K_{d}$ are the proportional, integral and derivative controller gains, respectively.

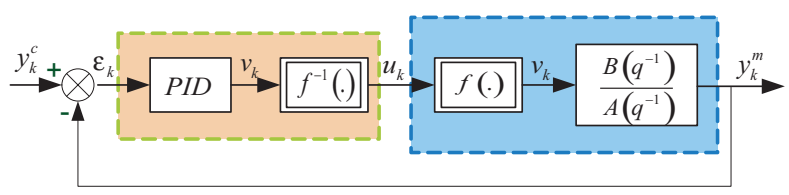

Fig. 2 - Nonlinear PID controller of a Hammerstein model [21].

This technique is valid only if the nonlinear fonction $f($. is invertible.

\section{B. Proposed PID controller based-on polynomial structure}

The proposed method consisted to approximate the inverse nonlinear gain using the polynomial structure, noted $f_{a p p}^{-1}($.$) .$ It eliminated the effect of the nonlinear gain in the Hammerstein model. Hence, a new nonlinear PID, noted PID ${ }^{N L}$, is obtained wish is described as follow:

$$
P I D^{N L}=P I D f_{a p p}^{-1}(.)
$$

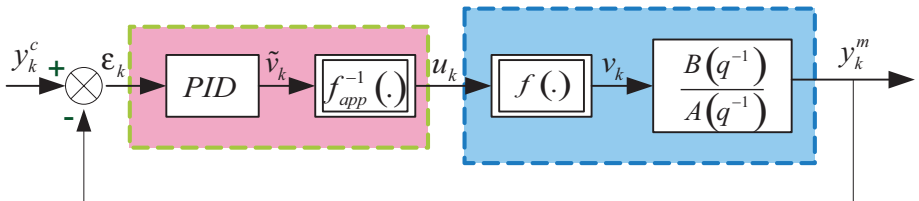

Fig. 3 - Proposed nonlinear PID controller of a Hammerstein model.

Noting that $\tilde{v}(k)$ is an approximate signal of $v_{k}$, we have chosen the following structure of $u_{k}=f_{a p p}^{-1}\left(\tilde{v}_{k}\right)$ :

$$
u_{k}=w_{1, k}^{3} V_{k}+\tilde{w}_{2, k}^{3} \tilde{V}_{k}^{[2]}+\tilde{w}_{3, k}^{3} \tilde{V}_{k}^{[3]}+O\left(V_{k}^{[4]}\right)
$$

$$
\begin{aligned}
& \text { with } V_{k}=\left(\begin{array}{l}
\tilde{v}_{k} \\
\tilde{v}_{k-1}
\end{array}\right), w_{1, k}^{3}=\left(\begin{array}{ll}
\beta_{1, k} & \beta_{2, k}
\end{array}\right) \text {, } \\
& \tilde{w}_{2, k}^{3}=\left(\begin{array}{lll}
\beta_{3, k} & \beta_{4, k} & \beta_{5, k}
\end{array}\right) \text { and } \tilde{w}_{3, k}^{3}= \\
& \left(\begin{array}{llll}
\beta_{6, k} & \beta_{7, k} & \beta_{8, k} & \beta_{9, k}
\end{array}\right) .
\end{aligned}
$$

By the identification of equations 8 and 13 , we obtain:

$$
\begin{aligned}
\beta_{1, k} & =1 ; \beta_{2, k}=-1 ; \beta_{3, k}=-\alpha_{1, k} ; \\
\beta_{4, k} & =2 \alpha_{1, k}-\alpha_{2, k} ; \beta_{5, k}=\alpha_{2, k}-\alpha_{3, k} ; \\
\beta_{6, k} & =2 \alpha_{1, k}^{2}-\alpha_{4, k} ; \\
\beta_{7, k} & =3 \alpha_{1, k} \alpha_{3, k}-6 \alpha_{1, k}^{2}+3 \alpha_{4, k}-\alpha_{5, k} ; \\
\beta_{8, k} & =-5 \alpha_{1, k} \alpha_{2, k}+5 \alpha_{1, k}^{2}+2 \alpha_{1, k} \alpha_{2, k}-3 \alpha_{4, k} \\
& -2 \alpha_{5, k}-\alpha_{6, k} ; \\
\beta_{9, k} & =\alpha_{4, k}+3 \alpha_{1, k}^{2}+\alpha_{1, k} \alpha_{2, k}+\alpha_{2, k} \alpha_{3, k}+3 \alpha_{5, k} \\
& +\alpha_{6, k} .
\end{aligned}
$$

\section{Illustrate Example: Coupled Two-Tank SYSTEM}

A two-tank system is used to illustrate the performance of the proposed polynomial structures of the Hammerstein model and the effectiveness of the proposed control approach.

\section{A. System description}

The system setup is a model of a chemical plant fragment. Very often tanks are coupled through pipes and the reactant level and flow has to be controlled. In the proposed work, only pump 1, tank 1, tank 2, and corresponding sensors have been used in SISO configuration, Fig. 4.

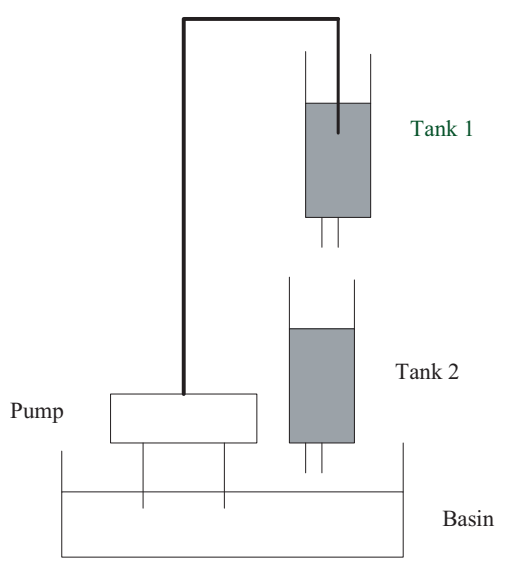

Fig. 4 - Diagram for two-tank system. 


\section{B. Mathematical modeling}

The coupled tank system is described by the following nonlinear equations:

$$
\left\{\begin{array}{l}
h_{1}=K \text { sat }\left(h_{11}\right) \\
\dot{h}_{11}=\eta \operatorname{sat}(u)-\frac{a_{1}}{A} \sqrt{2 g \operatorname{sat}\left(h_{11}\right)} \\
h_{2}=K \text { sat }\left(h_{22}\right) \\
\dot{h}_{22}=\frac{a_{1}}{A} \sqrt{2 g \operatorname{sat}\left(h_{11}\right)}-\frac{a_{2}}{A} \sqrt{2 g \operatorname{sat}\left(h_{22}\right)}
\end{array}\right.
$$

with:

$$
\begin{gathered}
\operatorname{sat}\left(h_{i i}\right)=\left\{\begin{array}{lll}
h_{i i} & \text { if } & 0 \leq h_{i i} \leq 0.3 \\
0.3 & \text { if } & h_{i i}>0.3 \\
0 & \text { if } \quad h_{i i}<0.3
\end{array} \text { for } i=1,2 .\right. \\
\text { sat }(u)=\left\{\begin{array}{lll}
u & \text { if } \quad 0 \leq u \leq 5 \\
5 & \text { if } & u>5 \\
0 & \text { if } & u<0
\end{array}\right.
\end{gathered}
$$

$h_{1}$ and $h_{2}$ denote the water level in the corresponding tank and $u$ is voltage applied to the pumps. $a_{1}$ and $a_{2}$ are the outlet area of the tanks, $\eta$ constant relating the control voltage with the water flow from the pump, $A$ is the cross-sectional area of the tanks and $g$ is the gravitational constant. The values of the simulink system parameters are shown in the table I.

TABLE I - Parameters of the System [25]

\begin{tabular}{cccc}
\hline & Value & Unit & Description \\
\hline$u$ & $0-5$ & $V$ & Voltage level of pump \\
$A$ & 0.01389 & $m^{2}$ & Cross-sectional area \\
$a_{i}$ & $50.26510^{-6}$ & $m^{2}$ & Outlet area of tank $i$ \\
$\eta$ & $2.410^{-3}$ & $\frac{m^{3}}{V \cdot s}$ & Water level of tank $i$ \\
$g$ & 9.81 & $m^{-2}$ & Gravitational constant \\
$K$ & 100 & -- & constant \\
\hline
\end{tabular}

\section{Parametric estimation and control result} using the exact inverse function of $f($.

The input of the system is shown in Fig. 5. The signal is set to $[0 \ldots+5 V]$. It's a pseudo-random binary sequence SBPA. The value of the sampling period is $T_{e}=1 s$.

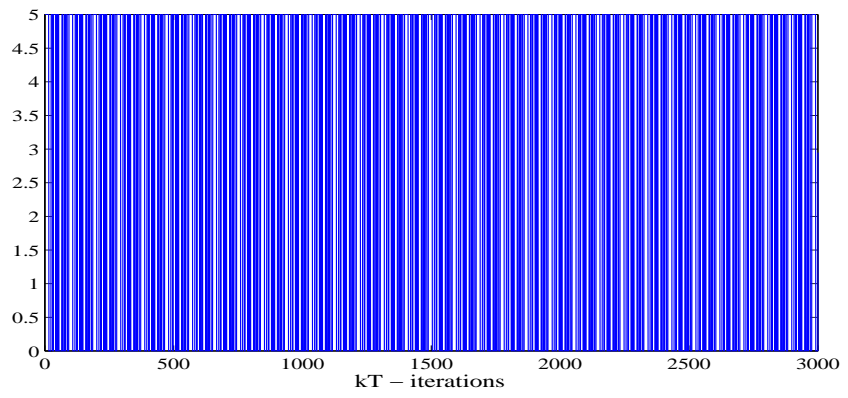

Fig. 5 - SBPA signal $u_{k}$.
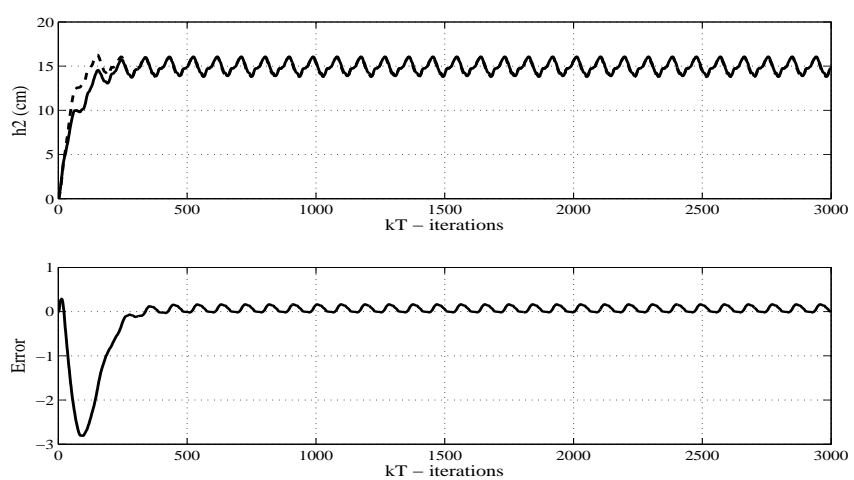

Fig. 6 - Responses of the real (solid line) and estimated (dotted line) output $h_{2}$.

Fig. 6. shows the responses of estimated and real output. Simulation results demonstrated that the error between the real system and the identified structures is negligible. The estimated model is described by:

$\left\{\begin{array}{l}y_{k}=\frac{B\left(q^{-1}\right)}{A\left(q^{-1}\right)} v_{k}=10^{-3} \frac{3.7633 q^{-1}+3.7748 q^{-2}}{1-1.9518 q^{-1}+0.9527 q^{-2}} v_{k} \\ v_{k}=f\left(u_{k}\right)=u_{k}+5 u_{k}^{2}\end{array}\right.$

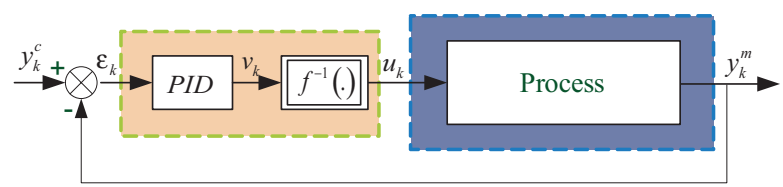

Fig. 7 - Nonlinear PID controller of a two-tank system.

Fig. 7 illustrates the diagram block of the nonlinear PID controller of a two-tank system. It consists of:

- the inverse of the nonlinearity: $u_{k}=f^{-1}\left(v_{k}\right)=0.1\left(\sqrt{1+20 v_{k}}-1\right)$, for $v_{k}>-0.05$

- the PID regulator:

$$
\left(K_{p}, K_{i}, K_{d}\right)=(4.5,1,0)
$$
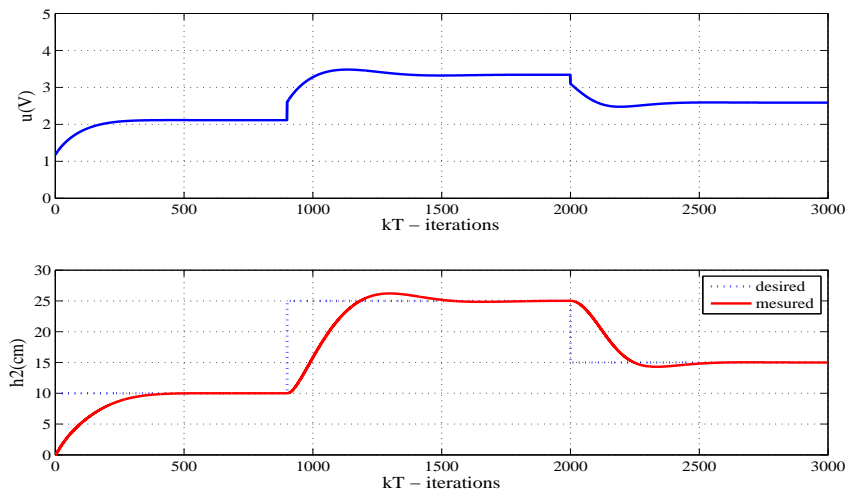

Fig. 8 - Responses of $u$ and $h_{2}$ of identified model. 

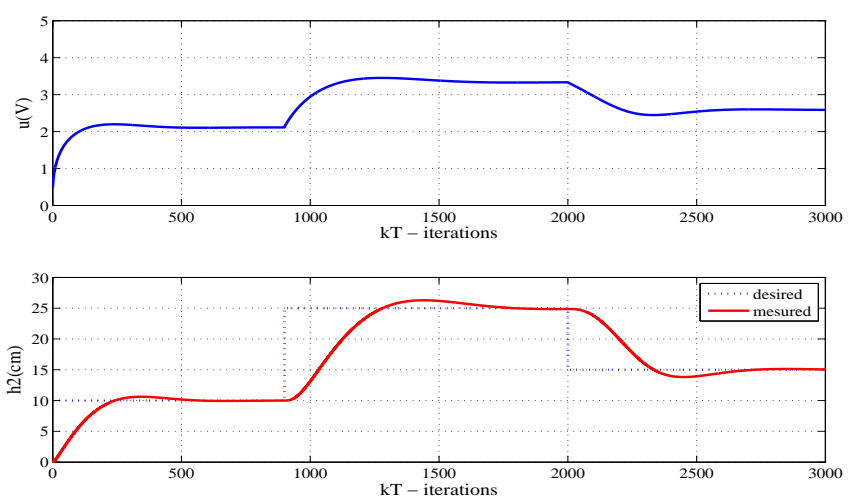

Fig. 9 - Responses of $u$ and $h_{2}$ of system.

Figures 8 and 9 show the control signals and the responses respectively of the mathematical model and the simulink system using the first method. Simulation results show that both follow the desired reference. To improve the results, we can increase the order of the polynomial $v_{k}=f\left(u_{k}\right)$. The problem is the no-existence of the inverse of the nonlinear function or the complexity of the computation of the inverse $f^{-1}\left(v_{k}\right)$.

\section{Parametric estimation and control result using polynomial} structures

We used the signal shown in Fig. 5 as input to estimate the model described by:

$$
\left\{\begin{array}{c}
y_{k}=\frac{B\left(q^{-1}\right)}{A\left(q^{-1}\right)} v_{k}=10^{-3} \frac{3.7657 q^{-1}+5.1097 q^{-2}}{1-1.9518 q^{-1}+0.9527 q^{-2}} v_{k} \\
v_{k}=\tilde{w}_{1, k}^{1} U_{k}+\tilde{w}_{2, k}^{1} \tilde{U}_{k}^{[2]}+\tilde{w}_{3, k}^{1} \tilde{U}_{k}^{[3]}+O\left(U_{k}^{[4]}\right)
\end{array}\right.
$$

with:

$$
\begin{gathered}
\tilde{w}_{2, k}^{1}=\left(\begin{array}{lll}
5 & -0.0571 & 5
\end{array}\right) \\
\tilde{w}_{3, k}^{1}=\left(\begin{array}{llll}
25 & -0.2856 & -0.2856 & 525
\end{array}\right)
\end{gathered}
$$
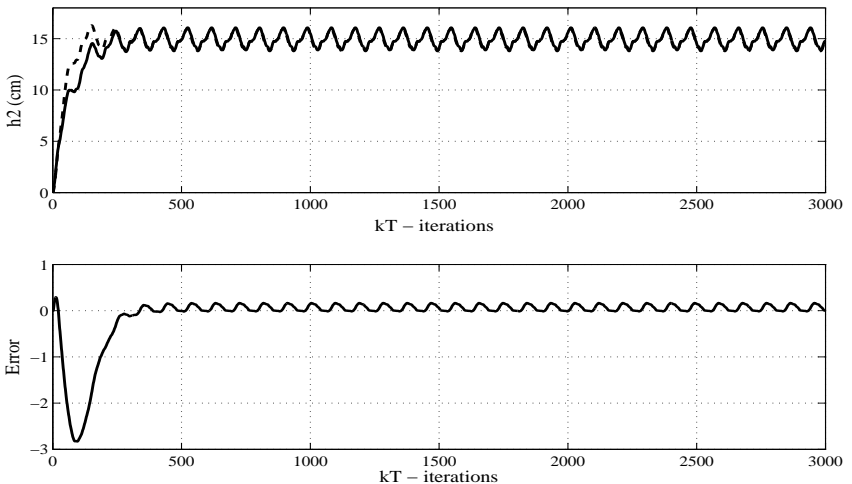

Fig. 10 - Responses of the real (solid line) and estimated (dotted line) output $h_{2}$ using the proposed approach.
Fig. 10 presents the responses of estimated and real output $h_{2}$. Simulation results demonstrate that the proposed structure describe very well the system behavior.

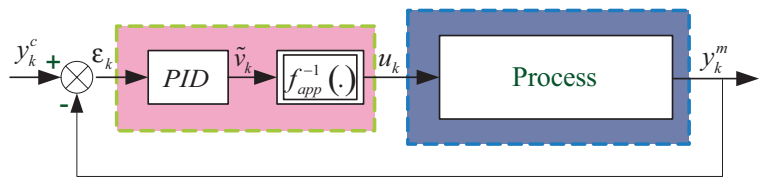

Fig. 11 - Proposed nonlinear PID controller of a two-tank system.

Fig. 11 shows the diagram block of the proposed nonlinear PID controller of a two-tank system. It is consists of:

- the inverse of the nonlinearity $f_{a p p}^{-1}\left(\tilde{v}_{k}\right)$ :

$$
u_{k}=w_{1, k}^{3} V_{k}+\tilde{w}_{2, k}^{3} \tilde{V}_{k}^{[2]}+\tilde{w}_{3, k}^{3} \tilde{V}_{k}^{[3]}+O\left(V_{k}^{[4]}\right)
$$

with:

$$
\begin{gathered}
w_{1, k}^{3}=\left(\begin{array}{ll}
1 & -1
\end{array}\right) \\
\tilde{w}_{2, k}^{3}=\left(\begin{array}{lll}
-5 & 10 & -5
\end{array}\right) \\
\tilde{w}_{3, k}^{3}=\left(\begin{array}{llll}
25 & 75 & 76 & 25
\end{array}\right)
\end{gathered}
$$

- the PID regulator:

$$
\left(K_{p}, K_{i}, K_{d}\right)=(0.45,0.7,0)
$$
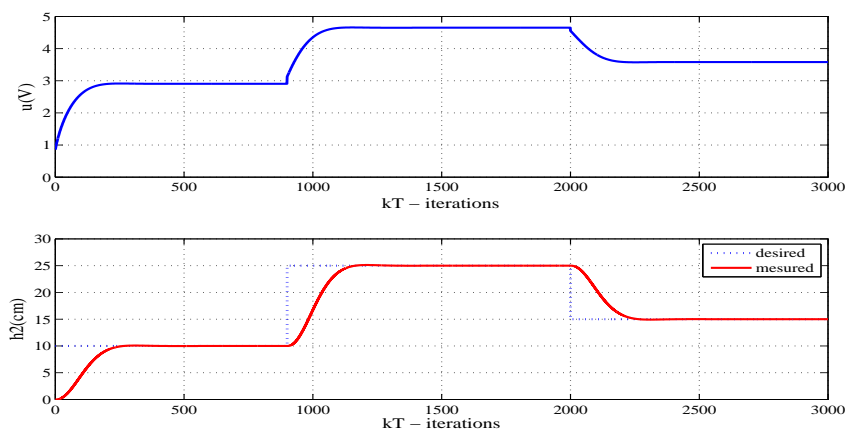

Fig. 12 - Responses of $u$ and $h_{2}$ using the proposed method of identified model.
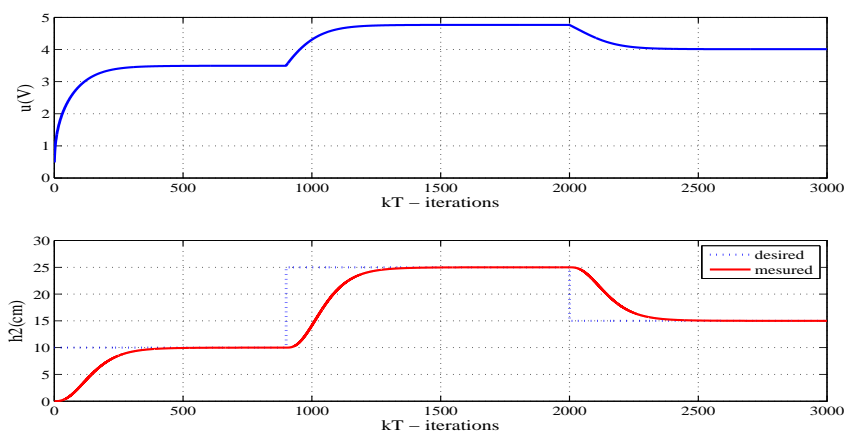

Fig. 13 - Responses of $u$ and $h_{2}$ using the proposed method of system. 
The control signals and the results of the regulator are presented in figures 12 and 13 . They proved that the $P I D^{N L}$ had achieved a satisfactory performance in tracking the reference signal.

\section{CONCLUSION}

In this paper, a new strategy to identify Hammerstein model has been proposed. A polynomial structure is used to model the nonlinear static function. This structure provide a good identification results. It has the advantage to approximate the inverse of the nonlinear part of Hammerstein model. A new design of $P I D^{N L}$ controller is successfully elaborated. It is composed by a PID associated with the inverted of the identified nonlinearity. A two-tank system is presented to illustrate the effectiveness of the proposed approach.

\section{REFERENCES}

[1] S. Lakshminarayanan, S. L. Shah and K. Nandakumar, "Identification of Hammerstein models using multivariate statistical tools". Chemical Engineering Science. vol. 50, pp. 3599-3613, 1995.

[2] N. Benhadj Braiek, H. Jribi and A. Becha, "A Technique of a Stability Domain Determination for Nonlinear Discrete Polynomial Systems". The International Federation of Automatic Contol, IFAC, pp. 8690-8694, 2008.

[3] N. Benhadj Braiek, "A Kronecker product approach of stability domain determination for nonlinear continuous systems". Journal of Systems Analysis Modelling and Simulation, ASMS, vol. 22, pp. 11-16, 1996.

[4] A. Novák, "Identification of Nonlinear Systems: Volterra Series Simplification". Acta Polytechnica, vol. 47, pp. 4-5, 2007.

[5] S. A. Billings and D. Coca, "Identification of NARMAX and related models". Research report-university of sheffield departement of automatic control and systems engieeng, 2001.

[6] K. P. Fruzzetti, A. Palazoglu and K. A. McDonald, "Nonlinear model predictive control using Hammerstein models". Journal of Process Control, vol. 7, pp. 31-41, 1997.

[7] E. Eskinat, S. H. Johnson and W. L. Luyben, "Use of Hammerstein models in identification of nonlinear systems". AIChE Journal, vol. 37, pp. 255268, 1991.

[8] R. K. Pearson, M. Pottmann and W. L. Luyben, "Gray-box identification of block-oriented nonlinear models". Journal of Process Control, vol. 10, pp. $301-315,2000$.

[9] H. T. Su, T. J. Mcavoy and W. L. Luyben, 'Integration of multilayer percepton networks and linear dynamic models: a Hammerstein modeling approach". Industrial and Engineering Chemistry Research, vol. 26, pp. 1927-1936, 1993

[10] W. M. Ling and D. E. Rivera, "Control relevant model reduction of volterra series models". Journal of Process Control, vol. 8, pp. 79-88, 1998.

[11] D. K Rollins, N. Bhanddari, A. M. Bassily, G. M. Colver and S. T. Chin, "A continuous-time nonlinear dynamic predictive modeling method for Hammerstein processes". Industrial and Engineering Chemistry Research, vol. 42, pp. 860-872, 2003.

[12] R. Mtar, M. M. Belhaouane, H. Belkhiria Ayadi and N. Benhadj Braiek, "An LMI criterion for the global stability analysis of nonlinear polynomia systems". Mathematical Modelling, Identification and Simulation vol. 9, pp. 171-183, 2009.

[13] I. K. Ibraheem and W. R. Abdul-Adheem, "On the Improved Nonlinear Tracking Differentiator based Nonlinear PID Controller Design". International Journal of Advanced Computer Science and Applications, vol. 1, pp. 234-241, 2016.

[14] G. P. Liu, V. Kadirkamanathan and S. A. Billings, "Predictive control for nonlinear systems using neural networks". International Journal of Control, vol. 71, pp. 1119-1132, 1998

[15] X. Hong, S. Iplikci, S. Chen and K. Warwick, "A model-based PID controller for Hammerstein systems using B-spline neural networks". International Journal of Adaptive Control and Signal Processing, vol. 28, pp. 412-428, 2014

[16] F. Chabni, R. Taleb, A. Benbouali and M. A. Bouthiba, The Application of Fuzzy Control in Water Tank Level Using Arduino". International Journal of Advanced Computer Science and Applications, vol. 7, pp. 261$265,2016$.
[17] A. R. Mcintosh, S. L. Shah and D. G. Fisher, "Analysis and tuning of adaptive generalized predictive control". The Canadian Journal of Chemical Engineering, vol. 69, pp. 97-110, 1991.

[18] T. Proll and M. N. Karim, "Real-time design of an adaptive nonlinear predictive controller". International Journal of Control, vol. 59, pp. 863889, 1994.

[19] F. G. Shinskey, "Process Control Systems". McGraw-Hill Book Company, New York, chapter 5, 1979.

[20] Y. Zhu, "Robust PID tuning using closed-loop identification". In Preprints of the International Symposium on Advanced Control of Chemical Processes ADCHEM, vol. 1, pp. 165-170, 2004.

[21] S. W. Sung, "System identification method for Hammerstein processes". Industrial and engineering chemistry research, vol. 41, pp. 4295-4302, 2002.

[22] E. Bai and M. Fu, "Identification of linear systems with hard input nonlinearities of known structure". IEEE Transactions on Signal Processing, vol. 38, pp. 853-860, 2002.

[23] F. Ding and T. Chen, "Identification of Hammerstein nonlinear ARMAX system". IEEE Transactions Automatic Control, vol. 41, pp. 1479-1489, 2005.

[24] J. Chainho, P. Pereira, S. Rafael and A. J. Pires, "A Simple PID Controller with Adaptive Parameter in a dsPIC; Case of Study". In Proceedings of the 9th Spanish-Portuguese Congress on Electrical Engineering, 2005.

[25] P. Roy, B. Kar, A. Kumar and B. K. Roy, "Level control of two tank system by fractional order integral state feedback controller tuned by PSO with experimental validation". In Control, Measurement and Instrumentation (CMI). IEEE First International Conference, 2016. 